\title{
Pigeon Pea Husk for Removal of Emerging Contaminants Trimethoprim and Atenolol from Water
}

\author{
Severin Eder ${ }^{1}{ }^{1}$, Manuel Torko ${ }^{1}$, Alessia Montalbetti ${ }^{1}$, Paride Azzari ${ }^{2} \mathbb{C}$ and Laura Nyström ${ }^{1, *}$ \\ 1 Laboratory of Food Biochemistry, Institute of Food, Nutrition and Health, Department of Health Science and \\ Technology, ETH Zurich, Schmelzbergstrasse 9, 8092 Zurich, Switzerland; severin.eder@hest.ethz.ch (S.E.); \\ mtorko@student.ethz.ch (M.T.); malessia@student.ethz.ch (A.M.) \\ 2 Laboratory of Food and Soft Materials, Institute of Food, Nutrition and Health, Department of Health Science \\ and Technology, ETH Zurich, Schmelzbergstrasse 9, 8092 Zurich, Switzerland; paride.azzari@hest.ethz.ch \\ * Correspondence: laura.nystroem@hest.ethz.ch; Tel.: +41-44-632-91-65
}

check for

updates

Citation: Eder, S.; Torko, M.;

Montalbetti, A.; Azzari, P.; Nyström,

L. Pigeon Pea Husk for Removal of

Emerging Contaminants

Trimethoprim and Atenolol from

Water. Molecules 2021, 26, 3158.

https://doi.org/10.3390/

molecules 26113158

Academic Editors: Urszula Guzik and Danuta Wojcieszyńska

Received: 29 April 2021

Accepted: 15 May 2021

Published: 25 May 2021

Publisher's Note: MDPI stays neutral with regard to jurisdictional claims in published maps and institutional affiliations.

Copyright: (c) 2021 by the authors. Licensee MDPI, Basel, Switzerland. This article is an open access article distributed under the terms and conditions of the Creative Commons Attribution (CC BY) license (https:// creativecommons.org/licenses/by/ $4.0 /)$.

\begin{abstract}
The pace of industrialization and rapid population growth in countries such as India entail an increased input of industrial and sanitary organic micropollutants, the so-called emerging contaminants (EC), into the environment. The emission of EC, such as pharmaceuticals, reaching Indian water bodies causes a detrimental effect on aquatic life and ultimately on human health. However, the financial burden of expanding sophisticated water treatment capacities renders complementary, cost-efficient alternatives, such as adsorption, attractive. Here we show the merits of washed and milled pigeon pea husk (PPH) as low-cost adsorbent for the removal of the EC trimethoprim (TMP) and atenolol (ATN) that are among the most detected pharmaceuticals in Indian waters. We found a linear increase in adsorption capacity of PPH for TMP and ATN at concentrations ranging from 10 to $200 \mu \mathrm{g} / \mathrm{L}$ and from 50 to $400 \mu \mathrm{g} / \mathrm{L}$, respectively, reflecting the concentrations occurring in Indian water bodies. Investigation of adsorption kinetics using the external mass transfer model (EMTM) revealed that film diffusion resistance governed the adsorption process of TMP or ATN onto PPH. Moreover, analysis of the adsorption performance of PPH across an extensive range of $\mathrm{pH}$ and temperature illustrated that the highest adsorption loadings achieved concurred with actual conditions of Indian waters. We anticipate our work as starting point towards the development of a feasible adsorbent system aiming at low-cost water treatment.
\end{abstract}

Keywords: low-cost adsorbent; side streams; pigeon pea; adsorption; emerging contaminants; trimethoprim; atenolol; adsorption kinetics; adsorption thermodynamics

\section{Introduction}

Worldwide access to clean water poses one of the greatest global challenges amidst a rapidly growing world population [1]. This endeavor becomes additionally complex due to the continuous deterioration of water quality caused by intensified anthropogenic activities in light of an increasing population [2]. Rapid industrialization, agricultural growth, and unstructured urbanization cause the entry of untreated sanitary waste, industrial effluents, and runoff from agricultural lands into water bodies and thus their contamination $[1,3]$. In this context, a broad group of organic micropollutants, the so-called emerging contaminants (EC), which were historically considered to be of minor concern given their distribution and concentration, have recently received particular attention. EC encompass substances belonging to the groups of pesticides, pharmaceuticals, personal care products, surfactants, and phthalates $[4,5]$. With the intensification of human activities and advances in analytical techniques, there is increasing awareness of EC and their adverse effects on the environment and human health.

India, as the fifth largest producer of pharmaceuticals and being among the largest consumer markets of drugs, faces significant issues with environmental inputs of pharmaceuticals, a major subgroup of EC [5,6]. Atenolol (ATN) and trimethoprim (TMP) represent 
one of the most frequently prescribed antihypertensives and antibiotics, respectively, and are among the most detected medical compounds in Indian water treatment plants. Concentrations in the range from 0.2 to $41.4 \mu \mathrm{g} / \mathrm{L}$ for ATN and from 0.003 to $4 \mu \mathrm{g} / \mathrm{L}$ for TMP have been reported in Indian water bodies $[5,6]$. Thus, the environmentally relevant concentration of both substances $(>1 \mu \mathrm{g} / \mathrm{L})$ is exceeded by multiple times in certain areas $[7,8]$. Their depositions into water reserves cause ecotoxicological effects on aquatic life and trigger antibiotic resistance in pathogens, which eventually presents a serious threat to human health [6,9].

However, the capacity of water treatment in India corresponds only to one third of the volumes of wastewater generated from its population. Moreover, the existing technology and infrastructure for water purification demonstrates rather limited effectiveness for the removal of pharmaceutical residues [4-6]. Yet, the nationwide extension with hightechnology systems is constrained due to immense investment expenses [1]. In this regard, adsorption presents an accessible, cost-efficient addition or alternative owing to the ease of operation and simplicity of design [2,10].

Recently, the replacement of activated carbon, the industrial standard, with cheaper alternatives based on agro-industrial side streams, the so-called low-cost adsorbents, has been pursued [2]. These waste materials possess a variety of functional groups and contribute to a reduction in unutilized plant by-products, thereby rendering their use attractive from several perspectives [11]. This vision has already been realized with the launch of the first household product to facilitate access to clean water in the Indian market. The Tata Swach system is an example of a successful application of low-cost adsorbents in a water purification device. The full-fledged, portable device, relies on rice husk ash as the adsorbent, and enables safe drinking water, in India, for low income households at an affordable price [12]. The establishment of a sustained, viable low-cost concept requires that a side stream material is readily available locally and in sufficient quantity. Pigeon Pea (Cajanus cajan) is the second most cultivated pulse in India and is mainly consumed as "Dal" $[13,14]$. For this, pigeon peas are dehulled and the cotyledon is split by an industrial process [11]. The production volume, in 2016, accounted for $2.8 \mathrm{Mt}$ in India, of which around $8 \%$, or approximately $230,000 \mathrm{t}$, account for husk material that end as side stream $[11,14]$. Consequently, pigeon pea husk (PPH) has potential to be utilized as a low-cost adsorbent; therefore, enhancing its exploitation. To date, adsorption studies with PPH have been limited to successful application for adsorption of various metals [11,15-17]. In contrast, the utilization of PPH for EC, such as pharmaceuticals, is still untapped.

Furthermore, many previous works included inconsistencies in their methodological approach and have used invalid assumptions for the process considered [18]. Numerous existing studies have based their investigations of adsorption thermodynamics on incorrect computations. An inappropriate derivation of the thermodynamic equilibrium constant $K_{e q}$ oftentimes results in unreasonable thermodynamic parameters [19]. Furthermore, the selection of isotherm models to describe the experimental isotherm data frequently relies on statistically incorrect criteria [20]. However, a valid modeling of the equilibrium data is essential for a meaningful evaluation of the adsorption process and the proper deduction of the adsorption isotherm parameters [10]. Moreover, the elucidation of adsorption kinetics is commonly performed by models that originate from the description of chemical reaction kinetics [21]. Therefore, these models fail to facilitate conclusive insight into the actual driving mechanisms of the mass transfer observed. The selection of adequate kinetic models with consideration of the characteristics of the adsorbent and the adsorption process is indispensable for a comprehensive assessment of the adsorption kinetics.

In this study, we evaluated the suitability of PPH as a low-cost adsorbent for the removal of the pharmaceutical substances, TMP and ATN, both present at elevated concentrations in Indian water bodies. For this purpose, we conducted a comprehensive analysis of the adsorption processes in a batch adsorption design which encompassed: (i) interpretation of the adsorption equilibrium, (ii) a detailed description of the adsorption kinetics, (iii) investigations of the thermodynamic parameters, and (iv) elucidation of the effects of 
temperature and $\mathrm{pH}$ on the adsorption performance. Particular attention was paid to the accurate derivation of the dimensionless thermodynamic equilibrium constants in order to obtain reasonable inferences about the nature of the adsorption process. In addition, we focused on numerical simulations of the adsorption kinetics with the external mass transfer model (EMTM) to validate the governing mechanism of the mass transfer. Furthermore, the selection of the appropriate isotherm model to predict the equilibrium data was based on the Akaike information criterion (AIC) methodology that provides a statistical criterion for the adequacy of considered candidate models. This study demonstrates that PPH poses a promising asset as a low-cost adsorbent for the removal of TMP and ATN and provides the scientific framework for the development of adsorption systems intended for EC with similar physiochemical properties.

\section{Results and Discussions}

\subsection{Surface Morphology and Physical Characteristics of Pigeon Pea Husk (PPH)}

The micrographs acquired displayed a distinct difference in the surface morphology of the inner and outer surfaces of the pigeon pea husk (PPH) particles (Figure 1A,B); however, both sides were characterized by the regularity of their respective patterns. The SEM investigations of the inner surface demonstrated a nonporous, wrinkled surface (Figure 1A). The undulating morphology was devoid of sharp edges and enlarged the external surface area. In contrast, the outer surface exhibited a homogeneous, smooth surface (Figure 1B). As on the inner surface, pronounced cavities were absent on the outer surface except for smaller cracks distributed across the surface in a somewhat ordered geometry. Generally, the micrographs recorded provided no indication of the presence of a considerable internal surface within PPH particles. Nitrogen sorption and BET analysis confirmed the negligible internal surface area and suggested that adsorption occurs at the external surface (Table 1). Furthermore, the physical properties of PPH are summarized in Table 1. For more information on the particle size distribution of the PPH material, see Supplementary Materials (Figure S1).

\subsection{Characterization of Surface Functionalities and Adsorption Process}

Fourier-transform infrared (FTIR) spectroscopy was used to characterize the structural functionalities of PPH and to confirm the adsorption of trimethoprim (TMP) or atenolol (ATN) onto PPH. The evaluation focused on the functional group region of the spectra before and after TMP or ATN adsorption on PPH to identify the presence of characteristic functionalities (Figure 1C,D). The IR spectra of PPH (Figure 1C,D) exhibited bands typical for plant fiber material, such as the broad peak centered at $3320 \mathrm{~cm}^{-1}$, attributed to intermolecular $\mathrm{H}$-bonded $\mathrm{O}-\mathrm{H}$ stretching vibrations of alcoholic groups in polysaccharides [22,23]. The medium, sharper band detected at $3675 \mathrm{~cm}^{-1}$ can be attributed to $\mathrm{O}-\mathrm{H}$ stretching vibrations of free alcohol groups. Furthermore, the signals from 2990 to $2880 \mathrm{~cm}^{-1}$ originated from $\mathrm{C}-\mathrm{H}$ stretch vibrations. The peak detected at $1740 \mathrm{~cm}^{-1} \mathrm{can}$ be assigned to $\mathrm{C}=\mathrm{O}$ stretching vibrations of carboxyl group functionalities. Moreover, the bands at 1620 and $1400 \mathrm{~cm}^{-1}$ are typically ascribed to the stretching of carboxyl groups $(\mathrm{O}=\mathrm{C}-\mathrm{O})[23,24]$. The peak, occurring at $2360 \mathrm{~cm}^{-1}$, originated from the presence of trace amounts of atmospheric $\mathrm{CO}_{2}$ during the measurement.

The spectra acquired for TMP (Figure 1C) exhibited bands characteristics for its structural features [25,26]. The sharp bands at 3470 and $3320 \mathrm{~cm}^{-1}$ were assigned to $\mathrm{N}-\mathrm{H}$ stretching vibration of primary aromatic amine groups. Furthermore, the shoulder peak centered at $3110 \mathrm{~cm}^{-1}$ corresponded to the $\mathrm{N}-\mathrm{H}$ bending vibration [22,25]. The bands in the region 3030-2810 $\mathrm{cm}^{-1}$ were attributed to $\mathrm{C}-\mathrm{H}$ stretch vibrations of the pyrimidine, benzyl, and methyl groups [26]. Moreover, signals ranging from 1670 to $1540 \mathrm{~cm}^{-1}$ and at $1510 \mathrm{~cm}^{-1}$ correlated to the aromatic ring stretch of $\mathrm{C}=\mathrm{C}$ vibrations. Bands from 1360 to $1210 \mathrm{~cm}^{-1}$ and the broad peak at $1130 \mathrm{~cm}^{-1}$ were ascribed to C-N stretching vibrations of the aromatic amine group and the $\mathrm{C}-\mathrm{O}$ stretching vibrations of ester moieties, respectively [22,26]. 
Similarly, ATN showed characteristic bands corresponding to its structural features (Figure 1D). The two peaks at 3350 and $3160 \mathrm{~cm}^{-1}$ corresponded to the $\mathrm{N}-\mathrm{H}$ stretching vibration of $\mathrm{N}-\mathrm{H}_{2}$ groups. It may be assumed that an expected band in the region from 3360 to $3310 \mathrm{~cm}^{-1}$, related to a secondary amine functionality, probably overlaps with the strong N-H stretching signal [22]. Bands in the region $2990-2820 \mathrm{~cm}^{-1}$ originated from alkyl C-H stretch vibrations and the strong peak at $1635 \mathrm{~cm}^{-1}$ was attributed to $\mathrm{H}$-bonded $\mathrm{C}=\mathrm{O}$ stretching vibration of a primary amid group. Additionally, bands from 1620 to $1580 \mathrm{~cm}^{-1}$ and the sharp signal at $1515 \mathrm{~cm}^{-1}$ were characteristic for the aromatic ring stretch of $\mathrm{C}=\mathrm{C}$ vibrations. Bands at 1415 and $1240 \mathrm{~cm}^{-1}$ were ascribed to the $\mathrm{O}-\mathrm{H}$ bending of the alcohol and $\mathrm{C}-\mathrm{O}$ stretching vibrations of the phenolic ester group, respectively [22].
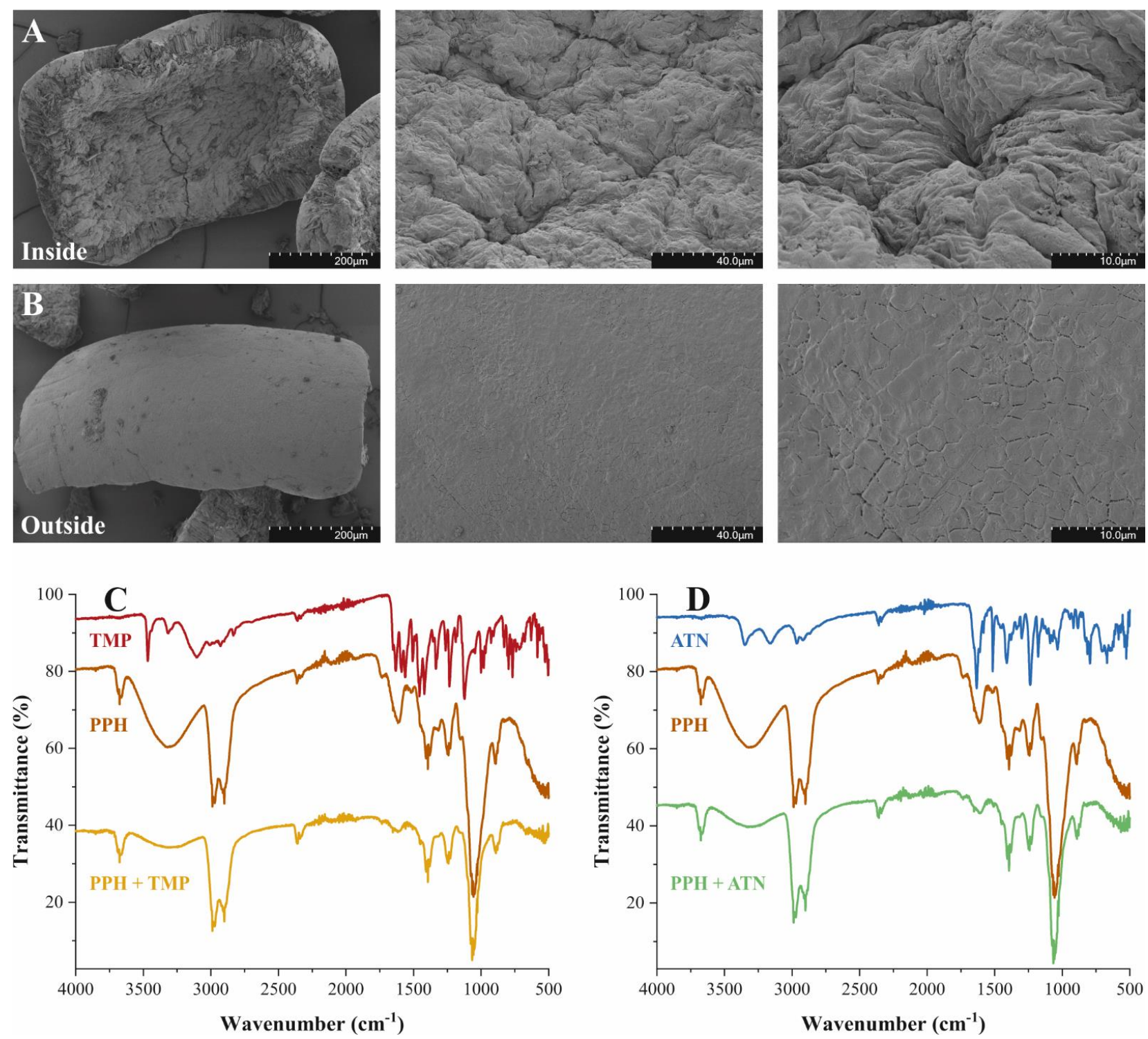

Figure 1. Representative SEM micrographs. (A) inside of PPH; (B) outside of PPH, at a series of magnifications. (C,D) FTIR spectra of TMP (-), ATN (-), PPH (-) and after adsorption of TMP (-) or ATN (-) on PPH.

A comparison before and after adsorption of TMP or ATN on PPH revealed an alteration or introduction of characteristic signals in the PPH spectra (Figure 1C,D). For instance, the broad signal of $\mathrm{O}-\mathrm{H}$ stretching vibrations at $3320 \mathrm{~cm}^{-1}$, noticeable in the PPH spectra, extenuated remarkably after adsorption of TMP. This attenuation might indicate the involvement of $\mathrm{O}-\mathrm{H}$ moieties, present on the $\mathrm{PPH}$, and aromatic amine 
groups, as part of the TMP structure, in the adsorption process. Additionally, the peak at $1450 \mathrm{~cm}^{-1}$, initially slightly pronounced in the PPH spectra, presented a characteristic signal after the adsorption experiment. Moreover, the bands ranging from 1690 to $1570 \mathrm{~cm}^{-1}$ significantly flattened in the spectra of PPH with adsorbed TMP as compared with the PPH spectra. Furthermore, the "fingerprint region" $<1000 \mathrm{~cm}^{-1}$ exhibited considerable variations, illustrating the presence of newly introduced functionalities by TMP on PPH (Figure 1C). The signal intensities at higher wave numbers decreased within the PPH spectra after ATN adsorption, as observed for TMP, suggesting that the corresponding functionalities participated in the adsorption (Figure 1D). A similar observation was made for the signals present in the PPH spectra ranging from 1700 to $1570 \mathrm{~cm}^{-1}$, indicating the presence of ATN on PPH after adsorption. The differentiation of the "fingerprint region" $<1000 \mathrm{~cm}^{-1}$ within the PPH spectra after the adsorption experiment corroborated the adsorption of ATN. The FTIR investigations of PPH before and after exposure to TMP or ATN revealed the accumulation of the respective substance on PPH and verified a successful adsorption process.

Table 1. Physical properties of PPH adsorbent material.

\begin{tabular}{cc}
\hline Physical Properties & PPH \\
\hline Specific surface area $\left(\mathrm{m}^{2} / \mathrm{g}\right)$ & 0.21 \\
Particle size $(\mu \mathrm{m})$ & 74 \\
Apparent density $\left(\mathrm{g} / \mathrm{cm}^{3}\right)$ & 0.72 \\
Solid density $\left(\mathrm{g} / \mathrm{cm}^{3}\right)$ & 1.5 \\
Particle porosity $(-)$ & 0.52 \\
\hline
\end{tabular}

\subsection{Adsorption Isotherm and Saturation Limit}

The adsorption equilibrium isotherms represented the adsorbed amount of TMP or ATN on PPH relative to the concentration of TMP or ATN in solution at equilibrium (Figure 2). Equilibrium experiments on the adsorption of TMP or ATN were conducted once in the $\mathrm{ppb}$ and once in the ppm concentration range. The adsorption isotherms acquired in the lower $\mathrm{ppb}$ range revealed insights into the adsorption process at TMP and ATN concentrations occurring in Indian water bodies (Figure 2A,B). Both adsorption systems were characterized by a linear relationship between PPH loading and TMP or ATN concentration. The Henry isotherm, suited for linear isotherms and commonly found at low concentrations, fitted the experimental data well $\left(R^{2}>0.97\right)$ (Figure 2A,B). The Henry constant, $K_{H}$, reflects the proportionality between the adsorbent loading and the concentration of TMP or ATN in solution. For both systems, the resulting $K_{H}$ values were greater than $1 \mathrm{~L} / \mathrm{g}$, illustrating a steeper increase in adsorption uptake with respect to a change in concentration.

The adsorption isotherm at a higher ppm concentration range enabled the determination of the maximum adsorption capacity of TMP and ATN on PPH (Figure 2C,D). The shape of the equilibrium isotherm reflects the affinity between adsorbate and adsorbent and facilitates insight into a possible adsorption mechanism associated with the interaction. According to the classification for liquid-solid adsorption systems, both adsorption isotherms observed can be identified as L curves [27]. The concave curves follow the assumption that a higher concentration of TMP or ATN results in higher adsorption capacity until the number of available adsorption sites become scarce. The increasing competition between TMP or ATN molecules for a vacant site results in the progressive saturation of PPH. Moreover, the L curve isotherm suggests that the interactions between TMP or ATN and $\mathrm{PPH}$, respectively, are driven by relatively weak forces. 

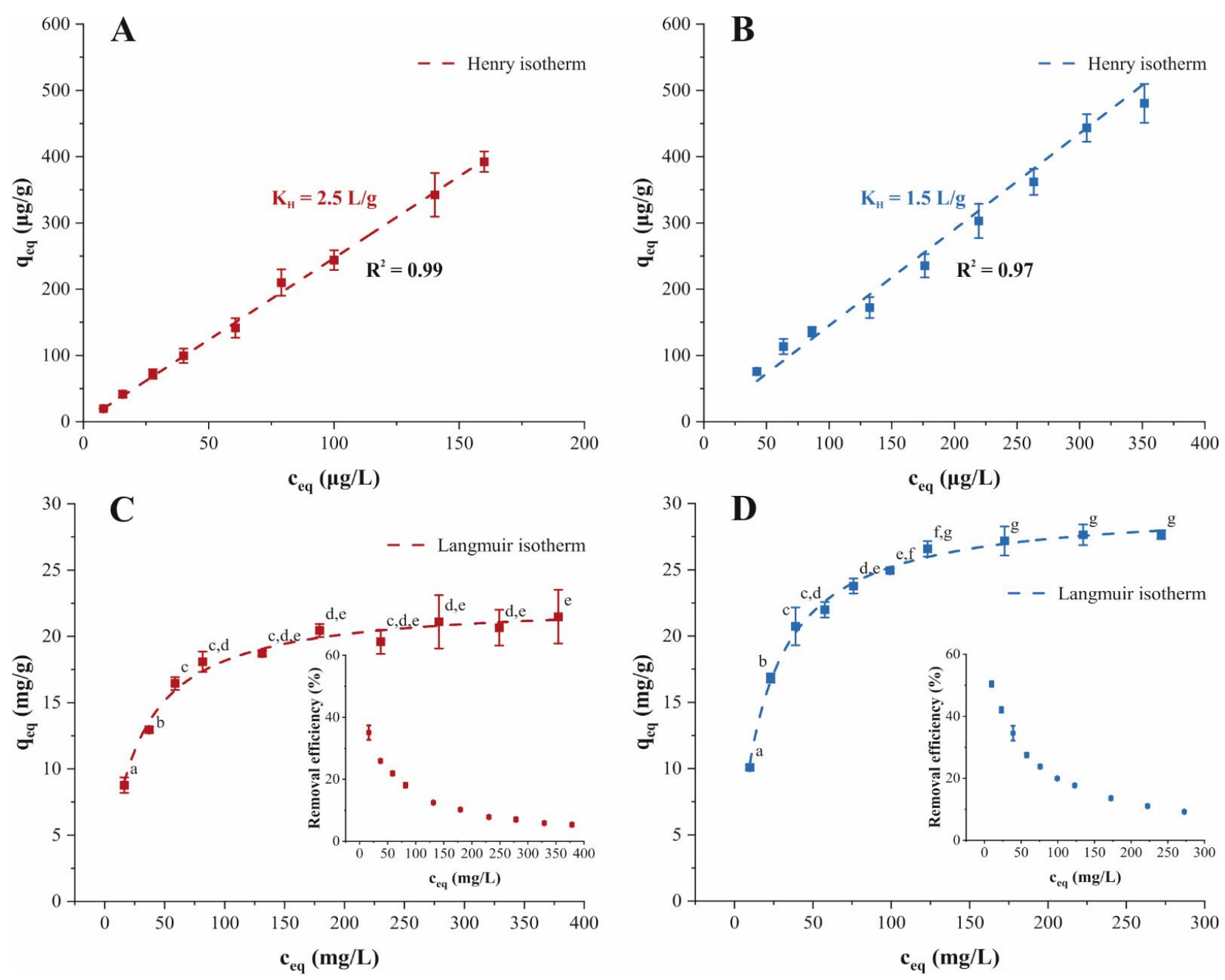

Figure 2. Experimental data ( $\square$ ) and Henry isotherm model (- - -) of the adsorption isotherms for (A) TMP (-) and (B) ATN adsorption (-) on PPH in the ppb concentration range. Experimental data ( $\square$ ) and Langmuir isotherm model (- - -) of the adsorption isotherms for (C) TMP (-) and (D) ATN adsorption (-) on PPH in the ppm concentration range. Different letters denote significant differences between isotherm points $(p<0.05, n=3)$.

We applied the Langmuir, Freundlich, Sips, Tóth, and Redlich-Peterson isotherm models to the equilibrium data of TMP and ATN adsorption to gain detailed insight into the nature of the adsorption process. According to the Akaike information criterion (AIC) methodology [28], we obtained a statistical comparison to determine the best model for the approximation of the experimental data. The isotherm model resulting in the highest $w_{i}$ value and lowest $\Delta_{i}$ value was deemed to be the best fit. The Langmuir isotherm presented the best approximation for TMP and ATN adsorption onto PPH, respectively (Table 2). Considering the $\mathrm{AIC}_{\mathrm{c}}$ values and statistical parameters derived thereof among models studied, the prediction of the experimental data for TMP adsorption ranked as follows: Langmuir $>$ Tóth $>$ Sips $>$ Redlich-Peterson $>$ Freundlich. In the case of ATN adsorption on $\mathrm{PPH}$, the isotherm models ranked as follows: Langmuir $>$ Tóth/Sips $>$ Redlich-Peterson $>$ Freundlich. Generally, isotherm models incorporating the saturation effect at high adsorbate concentration provided better fits than models approaching the Freundlich model. The $\Delta_{i}$ facilitates a relative strength of evidence comparison among candidate models and can be assessed following some simple rules, i.e., values of $\Delta_{i} \leq 2$ indicate considerable evidence for the model, models having $4 \leq \Delta_{i} \leq 7$ indicate substantially less support, and models with $\Delta_{i}>10$ have basically no evidence relative to the best model [28]. According to these guidelines, the Freundlich model possessed no evidence as a plausible model fit for either the adsorption of $\operatorname{TMP}\left(\Delta_{i}=26\right)$ or $\operatorname{ATN}\left(\Delta_{i}=56\right)$ onto PPH 
(Table 2). However, there was considerable strength of evidence for the Redlich-Peterson $\left(\Delta_{i}=2.5\right)$, Sips $\left(\Delta_{i}=1.8\right)$, and Tóth $\left(\Delta_{i}=1.7\right)$ isotherm models for TMP adsorption on PPH. Likewise, the three-parameter isotherms, Redlich-Peterson $\left(\Delta_{i}=2.5\right)$, Sips $\left(\Delta_{i}=2.4\right)$, and Tóth $\left(\Delta_{i}=2.4\right)$ showed substantial support as adequate predictions of the empirical data for ATN adsorption. The Akaike weights, $w_{i}$, provide a measure of the "weight of evidence", interpretable as probabilities, that a given model is indeed the best for the data obtained. The Langmuir model presented $w_{i}$ values of 0.43 and 0.57 for TMP or ATN adsorption on $\mathrm{PPH}$, expressible as $43 \%$ and $57 \%$ confidence in being the best approximation, respectively (Table 2). Owing to the high $w_{i}$ values, any possible model selection uncertainty could be eliminated. On the basis of $\Delta_{i}$, the evidence ratio $(E R)$ can be derived as an additional measure to reflect the relative likelihood between models. For the adsorption of TMP on $\mathrm{PPH}$, the ER obtained suggested that the Langmuir model was over $46 \times 10^{3}$ times more likely than the Freundlich model, two times more likely than the Sips and Tóth model, and three times more likely than the Redlich-Peterson model (Table 2). Accordingly, it was observed that the best-fitting Langmuir model was $1.2 \times 10^{12}$ times more likely than the Freundlich model, and three times more likely than the Sips, Tóth, and Redlich-Peterson models in the case of ATN adsorption on PPH (Table 2). Overall, the Freundlich model yielded poor model fits $(E R>150)$ for the adsorption processes considered [28].

Table 2. Results of model selection among five isotherm models based on AIC for adsorption equilibrium modeling and Langmuir parameters derived for TMP or ATN adsorption onto PPH.

\begin{tabular}{|c|c|c|c|c|c|c|c|}
\hline & Freundlich & $\begin{array}{l}\text { Redlich- } \\
\text { Peterson }\end{array}$ & Sips & Tóth & & Langmuir & \\
\hline & & & & & $\begin{array}{c}q_{m L} \\
(\mathrm{mg} / \mathrm{g})\end{array}$ & $K_{L}(\mathrm{~L} / \mathrm{mg})$ & $R_{L}$ \\
\hline TMP & & & & & 22.7 & 0.04 & $0.06-0.5$ \\
\hline K & 2 & 3 & 3 & 3 & & 2 & \\
\hline AIC & 33 & 9 & 9 & 9 & & 7 & \\
\hline $\mathrm{AIC}_{\mathrm{c}}$ & 34 & 10 & 10 & 10 & & 8 & \\
\hline$\Delta_{i}$ & 26 & 2.5 & 1.8 & 1.7 & & 0.0 & \\
\hline$w_{i}$ & $1.02 \times 10^{-6}$ & 0.14 & 0.19 & 0.20 & & 0.47 & \\
\hline \multirow[t]{2}{*}{$E R$} & 461643 & 3 & 2 & 2 & & 1 & \\
\hline & & & & & $\begin{array}{c}q_{m L} \\
(\mathrm{mg} / \mathrm{g})\end{array}$ & $K_{L}(\mathrm{~L} / \mathrm{mg})$ & $R_{L}$ \\
\hline ATN & & & & & 29.8 & 0.054 & $0.06-0.48$ \\
\hline$K$ & 2 & 3 & 3 & 3 & & 2 & \\
\hline AIC & 41 & -12 & -12 & -12 & & -14 & \\
\hline $\mathrm{AIC}_{\mathrm{c}}$ & 42 & -11 & -11 & -11 & & -14 & \\
\hline$\Delta_{i}$ & 56 & 2.5 & 2.4 & 2.4 & & 0.0 & \\
\hline$w_{i}$ & $4.4 \times 10^{-13}$ & 0.15 & 0.16 & 0.16 & & 0.53 & \\
\hline$E R$ & $1.2 \times 10^{12}$ & 3 & 3 & 3 & & 1 & \\
\hline
\end{tabular}

The Langmuir isotherm assumes the gradual filling of all possible adsorption sites without intermolecular interaction between adjacent adsorbate molecules, resulting in the formation of a monolayer on the solid surface [29,30]. The complete monolayer adsorption capacity, $q_{m L}$, reflects the saturation plateau. The binding constant, $K_{L}$, relates to the affinity of the adsorbate towards the adsorbent with increment of adsorbate. Hence, higher $K_{L}$ corresponds to a higher increase in adsorption capacity as $K_{L}$ reflects the initial slope of the equilibrium curve. The low $K_{L}$ values observed describe the smooth initial slope of the adsorption isotherm and reflect the rapid attainment of PPH saturation with increasing initial concentration of TMP and ATN (Table 2). Furthermore, the separation factor, $R_{L}$, derived from the isotherm data, permits inferences whether the considered adsorption process is favorable $\left(0<R_{L}<1\right)$, unfavorable $\left(R_{L}>1\right)$, or irreversible $\left(R_{L}=0\right)$ [30]. The $R_{L}$ values obtained for TMP and ATN adsorption on PPH ranged between 0 and 1 , confirming the favorable nature of the adsorption processes studied (Table 2). The 
maximum achievable adsorption loading obtained for TMP and ATN onto PPH were $22.7 \mathrm{mg} / \mathrm{g}$ and $29.8 \mathrm{mg} / \mathrm{g}$, respectively (Table 2).

The comparison of the $q_{m L}$ values observed with adsorption isotherms of TMP or ATN approximated with the Langmuir isotherm model facilitated a meaningful interpretation of the experimental values. The adsorption capacity of PPH for TMP and ATN was higher than for other low-cost adsorbents and even outperformed granular activated carbon (Table 3). As anticipated, higher achievable adsorption capacities were reported for biomass-derived activated carbonaceous adsorbents or specific clay minerals intended for the removal of TMP and ATN (Table 3). The economic benefits and minimal resource demand of PPH outweighs the inferior adsorption capacity and highlights the excellent performance of PPH as a sustainable low-cost adsorbent for TMP and ATN. Moreover, PPH removed over $35 \%$ of TMP and $50 \%$ of ATN at the lowest initial concentrations (Figure 2C,D). At the highest initial concentration of $400 \mathrm{ppm}$ TMP and 300 ppm ATN, PPH still exhibited removal efficiencies close to 10 and over $5 \%$, respectively.

Table 3. Comparison of maximum adsorption capacities for TMP or ATN with various adsorbates at neutral $\mathrm{pH}$ and $\mathrm{RT}$.

\begin{tabular}{ccccc}
\hline Adsorbent & Adsorbate & Isotherm Model & $\boldsymbol{q}_{\boldsymbol{m} L}(\mathbf{m g} / \mathbf{g})$ & Reference \\
\hline Wood chippings & TMP & Langmuir & 8.3 & {$[31]$} \\
Carbonized sewage sludge/fish waste & TMP & Langmuir & 90 & {$[32]$} \\
Montmorillonite KSF & TMP & Langmuir & 130 & {$[33]$} \\
PPH & TMP & Langmuir & 22.7 & This study \\
Kaolinite & ATN & Langmuir & 10.7 & {$[34]$} \\
Granular activated carbon & ATN & Langmuir & 18.8 & {$[35]$} \\
Activated palm kernel shell & ATN & Langmuir & 192 & {$[36]$} \\
PPH & ATN & Langmuir & 29.8 & This study \\
\hline
\end{tabular}

\subsection{Adsorption Kinetics}

The elucidation of the underlying mechanisms that determine the rate of adsorption are pivotal to the understanding of the mass transfer under investigation. The external mass transfer model (EMTM) used in this work assumed that film diffusion was the only rate-limiting mechanism for adsorption and omitted constraints due to intraparticle resistances $[21,37]$. The external mass transfer through the boundary layer is reflected by the external mass transfer coefficient or film diffusion coefficient $k_{F}$. Optimal $k_{F}$ values were obtained by matching the numerical solution of the EMTM to the experimental decay curves.

The EMTM approximation of the experimental kinetic curves for six initial concentrations for TMP and ATN, ranging from 20 to $200 \mu \mathrm{g} / \mathrm{L}$ and from 75 to $400 \mu \mathrm{g} / \mathrm{L}$, respectively, incorporated the optimal value of $k_{F}$ for each data set (Figure 3). The state of equilibrium was attained after $45 \mathrm{~min}$ for TMP adsorption and reached after $60 \mathrm{~min}$ for ATN adsorption on PPH (Figure 3). The EMTM predicted the kinetic data of TMP and ATN consistently, substantiating the governance of the adsorption rate only by film diffusion. Furthermore, $\mathrm{N}_{2}$ sorption experiments and BET analysis demonstrated negligible internal surface and corroborated the adequacy of the EMTM for the adsorption processes studied (Table 1).

The film diffusion coefficients, $k_{F}$, observed for TMP and ATN, ranged from 4.1 to $1 \times 10^{-3} \mathrm{~cm} / \mathrm{s}$ and from 0.8 to $0.3 \times 10^{-3} \mathrm{~cm} / \mathrm{s}$, respectively (Figure 3). Given the linear relationship of the equilibrium isotherm, the $k_{F}$ values for TMP and ATN were expected to remain constant across all initial concentrations studied. The experimental data are in line with this assumption, apart from the lowest initial concentration for both adsorbate substances (Figure 3). The deviations observed might arise due to the larger influence of experimental inaccuracies at lower concentrations. Constant $k_{F}$ values upon increasing initial concentration conform to the Henry isotherm theory, supposing that active sites on the adsorbent are available abundantly in relation to the adsorbate at sufficiently low concentrations. Accordingly, the external mass transfer resistances remained unchanged with increasing initial concentrations, as reflected in the $k_{F}$ values, within the concentration ranges considered for TMP and ATN. 
Overall, film diffusion as the controlling mechanism of the mass transfers observed, suggests the opportunity to influence the adsorption rate by modification of adsorption conditions [37]. For instance, an increase in stirrer velocity of the batch system diminishes the thickness of the boundary layer and eventually reduces the equilibrium time required. Therefore, the rate of film diffusion, reflected in increasing $k_{F}$ values, would increase under these conditions. Generally, a reduction in the adsorbent's particle size enhances the total surface area available for adsorption and further contributes to a reduction of the film diffusion resistance [37]. However, the adsorption process studied was not impeded due to constraints of the availability of sufficient adsorption sites, as previously discussed. Thus, any alteration of the particle size of PPH would be expected to result in an insignificant impact on the process. Ultimately, each intended application necessitates a critical assessment of benefits or disadvantages associated with the adsorption conditions considered to achieve an optimal compromise.
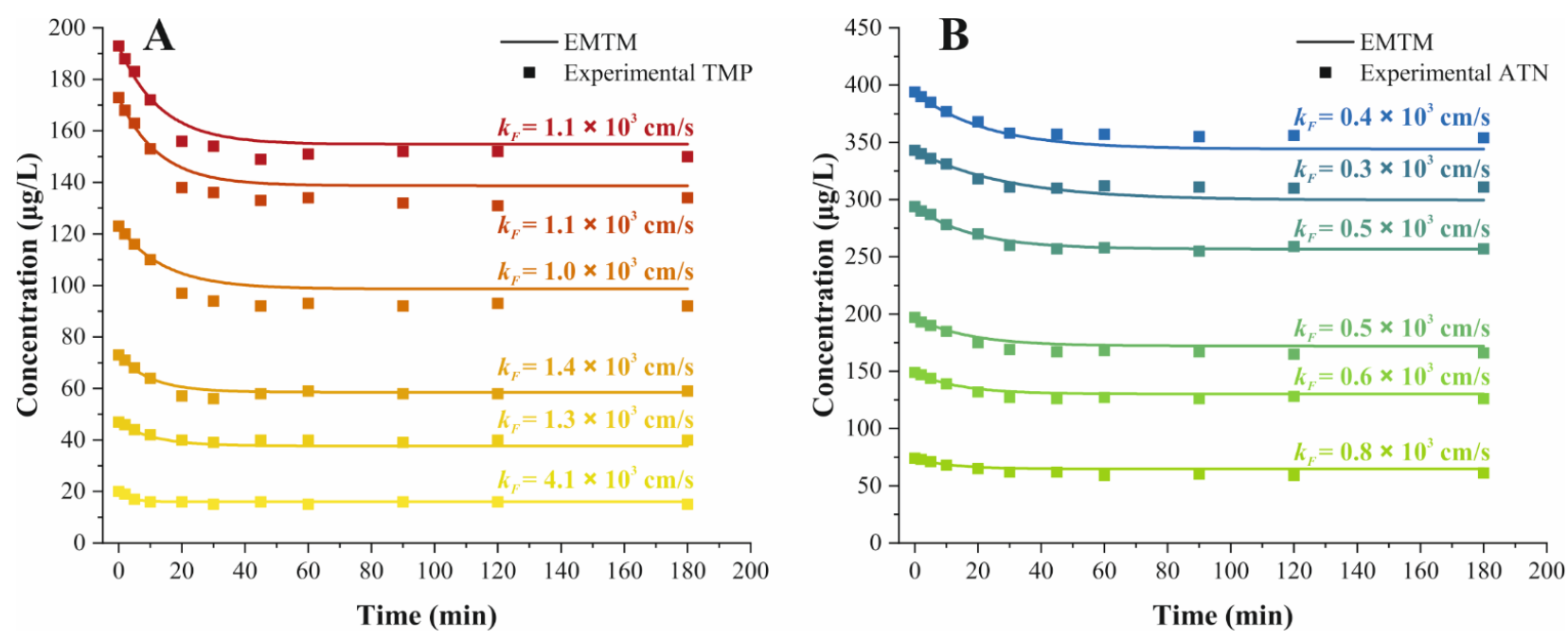

Figure 3. Experimental data ( $\mathbf{\square})$ and prediction of (A) TMP (-) and (B) ATN (-) decay curves with the EMTM during adsorption on PPH at six initial concentrations, respectively.

\subsection{Effect of Temperature and Adsorption Thermodynamics}

Temperature substantially affects the molecular interactions during an adsorption process, and therefore impacts the resulting equilibrium state. The effect of temperature on TMP and ATN adsorption on PPH was monitored at five temperatures analogous to the adsorption isotherms in the ppb range, respectively (see Section 2.3). Both adsorption systems studied showed increasing adsorption capacities with decreasing temperature from 333 to $277 \mathrm{~K}$, suggesting an exothermic adsorption process (Figure 4A,B). For instance, a temperature reduction from 333 to $277 \mathrm{~K}$ resulted in an increase in adsorption capacity from $211 \pm 29$ to $560 \pm 40 \mu \mathrm{g} / \mathrm{L}$ for TMP, and from $201 \pm 17$ to $630 \pm 50 \mu \mathrm{g} / \mathrm{L}$ for ATN adsorption on PPH at the highest initial concentration (Table S1). Overall, the adsorption capacity increased significantly from the highest to the lowest temperature for the respective initial concentrations of TMP or ATN considered (Figure 4A,B and Table S1). For a comprehensive overview with detailed information on significant differences between all temperature points for a respective initial concentration of TMP or ATN, see Supplementary Materials (Table S1).

The measurement of an adsorption isotherm at each temperature considered facilitated a meaningful derivation of the thermodynamic equilibrium constant $K_{e q}$ and a reliable elucidation of adsorption thermodynamics. The relevant thermodynamic parameters (Table 4) to assess the energetic conditions of the equilibrium states of the adsorption processes studied were derived from the Van't Hoff plot (Figure 4C,D). The negative standard free Gibbs energy $\left(\Delta G^{\circ}\right)$ values obtained indicated an exergonic process and showed the thermodynamically spontaneous nature of the adsorption (Table 4). Hence, 
the adsorption of TMP as well as ATN onto PPH presented a favorable process regardless of the solution temperature. Negative standard enthalpy $\left(\Delta H^{\circ}\right)$ values evidenced the exothermic nature of both adsorption processes as anticipated based on the effect of temperature described above (Table 4). The $\Delta H^{\circ}$ values observed for TMP and ATN adsorption suggested a physisorption controlled adsorption $\left(\Delta H^{\circ}<20 \mathrm{KJ}\right)$ driven by Van der Waals forces as fundamental interaction force [29]. These findings are in line with the interpretation of the isotherm curve for the determination of the maximum adsorption capacity (see Section 2.3). Considering the standard entropy $\left(\Delta S^{\circ}\right)$, positive values depicted the positive affinity of the adsorbents towards the adsorbate and the increase in disorder at the solid/liquid interface (Table 4). Furthermore, the dominant contribution of $\Delta H^{\circ}$, as compared with $T \Delta S^{\circ}$, to the negative $\Delta G^{\circ}$ values observed, indicated that both TMP and ATN adsorption on PPH were enthalpy governed processes [29,38].
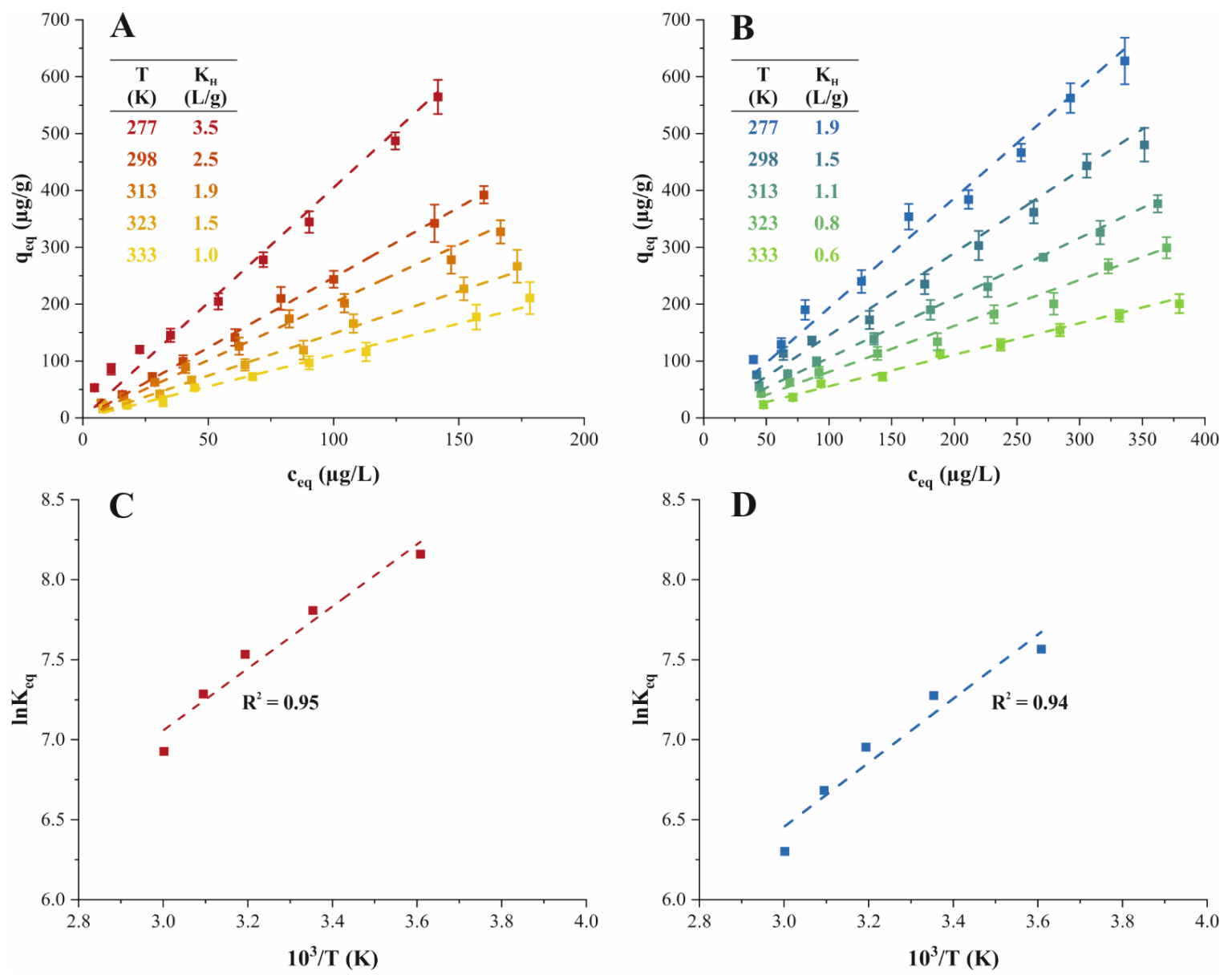

Figure 4. Experimental data (ם) and Henry isotherm model (- - -) of the effect of temperature on the adsorption performance of (A) TMP (-) and (B) ATN (-) on PPH. Van't Hoff plot and regression line (- - -) of (C) TMP (-) and (D) ATN (-) adsorption on PPH. Different letters denote significant differences between isotherm points $(p<0.05, n=3)$.

Table 4. Characteristic thermodynamic parameters of the adsorption process of TMP and ATN on PPH.

\begin{tabular}{cccccccc}
\hline & $\left.\boldsymbol{\Delta} \boldsymbol{H}^{\circ} \mathbf{( k J / m o l}\right)$ & $\boldsymbol{\Delta} \boldsymbol{S}^{\circ}(\mathbf{J} /(\mathbf{m o l} \times \mathbf{K}))$ \\
\hline & & & $277 \mathrm{~K}$ & $298 \mathrm{~K}$ & $313 \mathrm{~K}$ & $323 \mathrm{~K}$ & $333 \mathrm{~K}$ \\
TMP & -17.3 & 7.1 & -19.1 & -19.4 & -19.8 & -19.6 & -19.4 \\
ATN & -16.7 & 3.7 & -17.4 & -18 & -18.1 & -18 & -17.5 \\
\hline
\end{tabular}

\subsection{Effect of $p H$ on Adsorption Performance}

The adsorption behavior and the resulting adsorption capacity depend decisively on the $\mathrm{pH}$ of the solution (Figure $5 \mathrm{~A}, \mathrm{~B}$ ). Investigations of the effect of $\mathrm{pH}$ of the aqueous 
solution were performed at RT with $50 \mu \mathrm{g} / \mathrm{L}$ initial TMP and $150 \mu \mathrm{g} / \mathrm{L}$ initial ATN concentrations, respectively. As expected, the data obtained presented a strong dependance of initial solution $\mathrm{pH}$ and the adsorption loading of TMP or ATN on PPH (Figure 5A,B). The highest adsorption performance for TMP was observed at pH 6 with $105 \pm 7 \mu \mathrm{g} / \mathrm{g}$ (Figure 5A). In addition, PPH showed the maximum achievable loading for ATN at $\mathrm{pH} 8$ yielding $420 \pm 40 \mu \mathrm{g} / \mathrm{g}$ (Figure 5B). The adsorption capacities for both adsorbents decreased significantly at higher and lower $\mathrm{pH}$ conditions as compared with the respective point of maximum adsorption. In the case of TMP, the adsorption capacity achieved at $\mathrm{pH} 4$ was significantly lower as compared with the maximum adsorption at $\mathrm{pH}$ 6, but nevertheless, significantly higher than in the remaining $\mathrm{pH}$ points (Figure 5A). Furthermore, no significant difference in adsorption capacity was observed between $\mathrm{pH} 8$ and 10, whereas the adsorption loadings achieved at $\mathrm{pH} 2$ and $\mathrm{pH} 12$ were significantly lower than all other $\mathrm{pH}$ points studied for TMP. For adsorption of ATN on PPH, the data acquired at $\mathrm{pH} 6$ showed significantly inferior adsorption capacity as compared with $\mathrm{pH} 8$ but a significant increase towards the other $\mathrm{pH}$ points (Figure 5B). Moreover, the adsorption capacity of $\mathrm{ATN}$ at $\mathrm{pH} 10$ deviated significantly from $\mathrm{pH} 2$ and 12; however, no significant difference to $\mathrm{pH} 4$ was detected. The adsorption capacity at $\mathrm{pH} 2,4$, and 12 did not vary significantly. The functionalities of adsorbent and adsorbate, reflected in the point of zero charge (pzc) and $\mathrm{pK}_{\mathrm{a}}$ values, can explain the favorable or adverse interaction of adsorbent/adsorbate depending on the initial $\mathrm{pH}$ value (Figure $5 \mathrm{~A}, \mathrm{~B})$. TMP shows two $\mathrm{pKa}$ values $\left(\mathrm{pK}_{\mathrm{a} 1}=3.2\right.$ and $\mathrm{pK}_{\mathrm{a} 2}=7.1$ ), corresponding to the $\mathrm{pH}$ values at which the deprotonation of the respective amino group occurs [39]. Hence, TMP is present in its $\mathrm{TMP}^{2+}$ state for $\mathrm{pH}<3.2$, in its $\mathrm{TMP}^{+}$state for $3.2<\mathrm{pH}<7.1$, and as TMP for $7.1<\mathrm{pH}$. Similarly, ATN occurs in is protonated state $\mathrm{ATN}^{+}$below its pkA of 9.4 [40]. The pzc of PPH is defined by all functional groups on its surface and was determined to be at $\mathrm{pH}=5$ (Figure $\mathrm{S} 2$ ). At $\mathrm{pH}<\mathrm{pzc}$, the $\mathrm{PPH}$ carries a net positive charge and, at $\mathrm{pH}>\mathrm{pzc}$, the surface of $\mathrm{PPH}$ has a net negative charge.
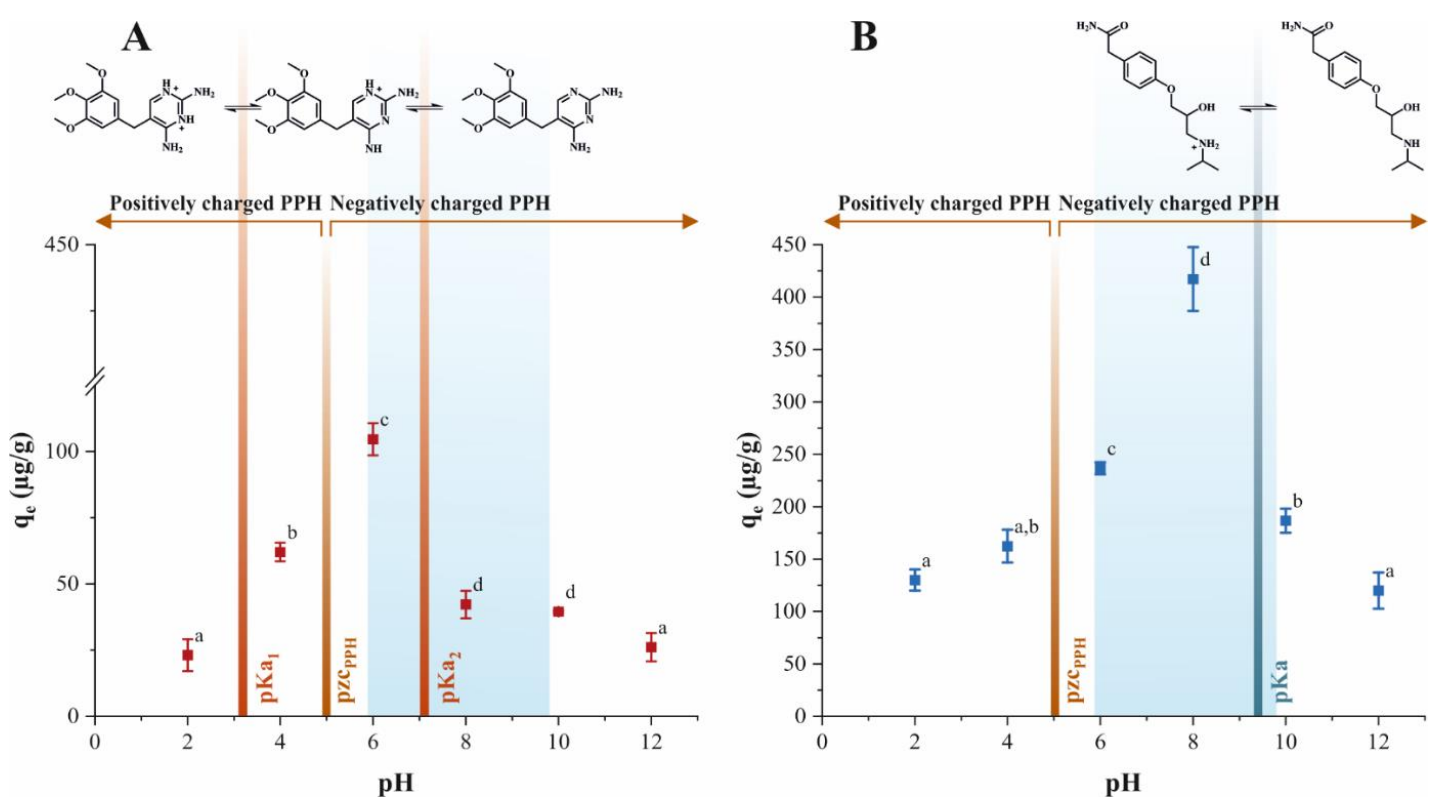

Figure 5. Effect of solution $\mathrm{pH}$ on the adsorption performance of (A) TMP ( $\square$ ) and (B) ATN ( $\square$ ) on PPH. The pH range of Indian water bodies ( $\mathrm{pH}$ 5.9-9.8) is highlighted with the shadowed background. Different letters denote significant differences between experimental points $(p<0.05, n=3)$.

The intersecting $\mathrm{pH}$ ranges where TMP or ATN exhibits a positive charge and $\mathrm{PPH}$ carries a negative surface charge corresponds to the $\mathrm{pH}$ points at which the highest adsorption charges were achieved, respectively. The effect of $\mathrm{pH}$ revealed the influence of PPH surface functionalities, which were elucidated within FTIR investigations, on the increase in attraction forces between adsorbate/adsorbent. Moreover, the $\mathrm{pH}$ values of 
the highest achievable adsorption loadings coincide with the $\mathrm{pH}$ range of Indian water bodies ( $\mathrm{pH}$ 5.9-9.8) [41-43], substantiating PPH as an adequate low-cost adsorbent for the application studied (Figure 5A,B). Overall, the data acquired provides a strong starting point for broader applications of PPH as low-cost adsorbents targeted at the removal of pharmaceuticals or emerging contaminants with similar physicochemical properties within the $\mathrm{pH}$ range considered.

\section{Materials and Methods}

\subsection{Chemicals}

Trimethoprim ( $\geq 99 \%)$, atenolol $(\geq 98 \%)$, formic acid $\left(\mathrm{CH}_{2} \mathrm{O}_{2}, 98-100 \%\right)$, potassium bromide $(\mathrm{KBr},>99 \%)$, and sodium hydroxide $(\mathrm{NaOH}, \geq 98 \%)$ were obtained from SigmaAldrich (St. Louis, MO, USA). Hydrochloric acid $(\mathrm{HCl},>37 \%)$ was purchased from VWR International (Radnor, $\mathrm{PA}, \mathrm{USA})$. Sodium chloride $(\mathrm{NaCl}, \geq 99.5 \%)$ and acetonitrile $\left(\mathrm{C}_{2} \mathrm{H}_{3} \mathrm{~N}\right.$, $\geq 99.9 \%$ ) were purchased from Fisher Scientific (Waltham, MA, USA). All solutions were prepared with purified water using a Millipore MilliQ system (Billerica, MA, USA).

\subsection{Preparation of Pigeon Pea Husk (PPH) Adsorbent Material}

Pigeon pea husk (PPH) was provided by Bühler AG (Uzwil, Switzerland). The PPH was sieved through a stack of sieves, consisting of 4 and $2 \mathrm{~mm}$ mesh size, to remove unwanted material. Subsequently, the husk material was sieved with $1 \mathrm{~mm}$ mesh size to separate pigeon pea residues and smaller particles. Any remaining impurities were removed manually. Afterwards, the PPH was ground and sieved with a ZM200 ultracentrifugal mill (Retsch $\mathrm{GmbH}$, Haan, Germany) to obtain a particle size $<80 \mu \mathrm{m}$. The powdered PPH was washed to avoid leaching and discoloration of the solution during adsorption experiments. For this purpose, PPH was added to water in a 1:20 solid/solution ratio $(v / v)$ and heated at $80{ }^{\circ} \mathrm{C}$ for 20 min under continuous agitation. After cooling, the suspension was centrifuged at $24 \times 10^{3} \mathrm{~g}$ for $10 \mathrm{~min}$ in a Sorvall LYNX 4000 Superspeed Centrifuge (Thermo Fisher Scientific, Waltham, MA, USA). The colored supernatant was discarded, the PPH material was redissolved in water, and the procedure repeated five times in total. Eventually, the PPH was dried in a hot air oven at $60^{\circ} \mathrm{C}$ overnight. PPH was stored in a desiccator under $\mathrm{N}_{2}$ atmosphere until further use.

\subsection{Characterization of PPH and Functional Investigations}

Micrographs of PPH were obtained using a SU5000 (Hitachi, Tokyo, Japan) highresolution field emission scanning electron microscope (SEM). Second electron (SE) signal was acquired at $2.0 \mathrm{kV}$ and a working distance of $5.5 \mathrm{~mm}$. Samples were sputter coated with $4.5 \mathrm{~nm}$ platinum-palladium prior to the collection of micrographs. The particle size distribution of PPH powder was determined with a Partica LA-950 Laser Diffraction Particle Size Distribution Analyzer (Horiba, Kyoto, Japan). The solid density $\left(\rho_{s}\right)$ of the PPH was analyzed utilizing an AccuPyc 1330 He-Pycnometer (Micromeritics, Norcross, GA, USA). Hg-Porosimetry (Pascal 140/440 Hg-Porosimeter, Thermo Fisher Scientific, Waltham, MA, USA) was used to determine the apparent density $\left(\rho_{p}\right)$ of the PPH. The particle porosity $\left(\varepsilon_{p}\right)[44]$ of the PPH was calculated according to:

$$
\varepsilon_{p}=1-\frac{\rho_{p}}{\rho_{s}} .
$$

Fourier-transform infrared (FTIR) spectroscopy analyses of PPH were conducted before and after adsorption experiments with TMP and ATN to infer about an effective adsorption. The FTIR spectra were recorded with a Jasco FT/IR-4100 spectrometer (JASCO Corporation, Tokyo, Japan) equipped with an Attenuated Total Reflectance (ATR) sampling accessory (ATR PRO ONE, JASCO Corporation, Tokyo, Japan) working with a monolithic diamond crystal. Spectra were recorded in the spectral range of $4500-500 \mathrm{~cm}^{-1}$ and processed using the Jasco Spectra Manager Version 2.05.02 (JASCO Corporation, Tokyo, Japan). 


\subsection{Batch Adsorption Design}

The potential of PPH as an adsorbent for trimethoprim (TMP) or atenolol (ATN) was assessed with respect to the effect of initial adsorbent concentration, contact time, temperature, and $\mathrm{pH}$. The batch adsorber design was chosen for the investigation of the adsorption systems, varying the parameter under investigation while all other parameters were kept constant. Equilibrium experiments were conducted at room temperature and neutral $\mathrm{pH}$ with an adsorbent dosage of $0.005 \mathrm{~g}$, a solution volume of $0.05 \mathrm{~L}$, and an initial concentration of TMP and ATN ranging from 10 to $200 \mu \mathrm{g} / \mathrm{L}$ and from 50 to $400 \mu \mathrm{g} / \mathrm{L}$, respectively. The considered concentration ranges reflected a compromise between analytical viability and the simulation of residue concentrations occurring in Indian water bodies. The adsorption system was kept in contact for $180 \mathrm{~min}$ and constantly agitated at $300 \mathrm{rpm}$ in a Unimax 1010 shaker (Heidolph, Schwabach, Germany). Adsorption kinetics were evaluated using six initial concentration for both adsorbates. The concentration decay over time was investigated with altered experimental conditions. PPH dose and solution volume were increased to $0.01 \mathrm{~g}$ and $0.1 \mathrm{~L}$, respectively, to prevent distortion of the solid/liquid ratio due to aliquots retrieved at preset time points. The effect of temperature on the resulting adsorption capacities for TMP or ATN was analyzed over a temperature range from 4 to $60^{\circ} \mathrm{C}$. The effect of initial $\mathrm{pH}$ on the achievable adsorption loading was monitored over a $\mathrm{pH}$ range from 2 to 12. The $\mathrm{pH}$ of the solution was adjusted with $\mathrm{HCl}$ or $\mathrm{NaOH}$ at appropriate concentrations to minimalize volume distortion. Furthermore, equilibrium experiments were performed at higher concentration ranges of the adsorbates to determine the maximum achievable saturation limit of PPH for TMP or ATN. For this purpose, an adsorbent dose of $0.01 \mathrm{~g}$, a solution volume of $0.01 \mathrm{~L}$, and initial concentrations ranging from 25 to $400 \mathrm{mg} / \mathrm{L}$ for TMP and from 20 to $300 \mathrm{mg} / \mathrm{L}$ for ATN were selected.

After incubation, the mixtures were centrifuged for $15 \mathrm{~min}$ at $4000 \mathrm{rpm}$. Subsequently, the remaining TMP or ATN concentration in the liquid phase was determined using reversed-phase ultra-performance liquid chromatography with diode array detection (RPUHPLC-DAD) (see Section 3.5). The adsorption uptake of TMP or ATN on PPH can be derived from the mass balance equation [30], which is given for any point of the adsorption process by:

$$
q_{t}=\frac{\left(c_{0}-c_{t}\right) V}{W}
$$

where $c_{0}$ and $c_{t}$ depict the initial adsorbate concentration in solution $(\mu \mathrm{g} / \mathrm{L}$ or $\mathrm{mg} / \mathrm{L})$ and at any given time point $(\mu \mathrm{g} / \mathrm{L}$ or $\mathrm{mg} / \mathrm{L})$, respectively; $q_{t}$ reflects the adsorbate concentration on PPH at given time point ( $\mu \mathrm{g} / \mathrm{g}$ or $\mathrm{mg} / \mathrm{g}$ ); $V$ and $W$ correspond to the volume of the solution (L) and the weight of PPH $(\mathrm{g})$ used in the batch adsorption experiment, respectively.

\subsection{Determination of Trimethoprim and Atenolol Concentration}

The concentration of TMP and ATN in solution was analyzed by RP-UPLC-DAD For the analysis of residual adsorbate concentration, an Agilent 1290 Infinity II System (Agilent Technologies, Santa Clara, CA, USA) equipped with an Acquity UPLC BEH C8 $(2.1 \times 100 \mathrm{~mm}, 1.7 \mu \mathrm{m})$ column operating at $30^{\circ} \mathrm{C}$ was used. The method was adapted from the work by Krisko et al. [45], with slight modifications. The injection volume of the samples was $10 \mu \mathrm{L}$ and a combination of the two mobile phases (A) water and (B) acetonitrile, containing $0.1 \%$ formic acid $(v / v)$, was applied for elution at a flow rate of $0.4 \mathrm{~mL} / \mathrm{min}$. The resulting gradient program was $0-5 \mathrm{~min}$, from $99.5 \% \mathrm{~A}$ and $0.5 \% \mathrm{~B}$ linear to $95 \% \mathrm{~A}$ and $5 \% \mathrm{~B} ; 5-7 \mathrm{~min}$, linear to $85 \% \mathrm{~A}$ and $15 \% \mathrm{~B} ; 7-10 \mathrm{~min}$, linear to $5 \% \mathrm{~A}$ and $95 \% \mathrm{~B} ; 10-10.5 \mathrm{~min}$, isocratic $5 \% \mathrm{~A}$ and $95 \% \mathrm{~B} ; 10.5-11 \mathrm{~min}$, linear to $95 \% \mathrm{~A}$ and $5 \%$ B; $11-12$ min, linear to $99.5 \%$ A and $0.5 \%$ B. Detection of TMP and ATN was conducted at $272 \mathrm{~nm}$. Mixtures of TMP and ATN at seven concentration levels of $0.01-0.25 \mathrm{mg} / \mathrm{L}$ and $0.05-0.4 \mathrm{mg} / \mathrm{L}$, respectively, were used as external standards. For the determination of maximal adsorption loading, TMP and ATN at seven concentration levels of $0.25-20 \mathrm{mg} / \mathrm{L}$ and $0.5-50 \mathrm{mg} / \mathrm{L}$, respectively, were used as external standards. Quantification was based 
on the external calibration method using the Chromeleon Chromatography Data System (CDS) Version 7 (Thermo Fisher Scientific, Waltham, MA, USA).

\subsection{Adsorption Isotherm Models}

The interpretation of the adsorption equilibrium presents an essential procedure in the investigation of a considered adsorption process. Linear equilibrium relationships were described using the Henry isotherm and fitted by linear regression to derive the isotherm parameter. The Henry isotherm is defined as:

$$
q_{e q}=K_{H} c_{e q},
$$

where $q_{e q}$ and $c_{e q}$ are the equilibrium concentration in solution $(\mu \mathrm{g} / \mathrm{L})$ and on the adsorbent $(\mu \mathrm{g} / \mathrm{g})$, respectively. The equilibrium constant, known as the Henry constant $\mathrm{K}_{\mathrm{H}}(\mathrm{L} / \mathrm{g})$, equals the constant of proportionality, and reflects the relationship between liquid and solid phase concentration [29,30]. Equilibrium data, exhibiting a nonlinear course of the relationship, were analyzed using the Langmuir model, which is given by:

$$
q_{e q}=\frac{q_{m L} K_{L} c_{e q}}{1+K_{L} c_{e q}}
$$

where $q_{e q}$ and $c_{e q}$ were defined earlier and $q_{m L}$ depicts the Langmuir constant related to the monolayer adsorption capacity $(\mathrm{mg} / \mathrm{g})$. The Langmuir constant $K_{L}$ reflects the affinity of binding sites and the free energy of sorption $(\mathrm{L} / \mathrm{mg})$ [29]. The nature of the Langmuir isotherm can be characterized by a dimensionless constant separation factor $R_{L}$, which results from:

$$
R_{L}=\frac{1}{1+K_{L} c_{0}}
$$

Specific values of $R_{L}$ can be attributed to the isotherm characteristics, being either favorable $\left(0<R_{L}<1\right)$, unfavorable $\left(R_{L}>1\right)$, linear $\left(R_{L}=1\right)$, or irreversible $\left(R_{L}=0\right)$ [30]. Moreover, the Freundlich, Redlich Peterson, Sips, and Tóth isotherm models were applied to nonlinear adsorption isotherms. Here, the experimental data was fitted by nonlinear regression to the candidate isotherms. The model parameters were obtained by minimizing the sum of squared errors between predicted $\left(q_{c a l}\right)$ and experimental data $\left(q_{\exp }\right)$. The sum of squared errors is given by:

$$
S E=\sum_{i=1}^{N}\left(q_{c a l}-q_{\exp }\right)_{i}^{2}
$$

In either case, a plot of $c_{e q}$ versus $q_{e q}$ generated the experimental isotherm curves. For a detailed description of the adsorption isotherm models, see Supplementary Materials.

\subsection{Isotherm Model Selection for Adsorption Equilibrium Description}

The Akaike information criterion evaluates the relative adequacy of models to predict the experimental data, and thus provides a measure for the selection of the best-fit model [28]. The statistical parameters of the AIC necessary for model selection are briefly presented hereafter. A more detailed description and their mathematical derivation is given elsewhere [28]. The AIC value can be generally defined as:

$$
\mathrm{AIC}=-2 \ln (L)+2 \mathrm{~K}
$$

where $L$ is the maximized likelihood function for the respective model and $K$ is the number of parameters in this model. 
Assuming that the model errors are normally and independently distributed, and incorporating the least squares estimation, the AIC transforms to:

$$
\mathrm{AIC}=N \ln \left(\frac{S S E}{N}\right)+2 \mathrm{~K}
$$

where $N$ is the number of observations and SSE is the sum of squared errors.

The SSE is calculated analogous to Equation (6) for the difference between predicted $\left(q_{\text {eq }}, \mathrm{cal}\right)$ and experimental adsorbent loading $\left(q_{\text {eq,exp }}\right)$ at equilibrium. For the case that $K$ is large relative to $N(N / K<40)$, Sugiura [46] conceived the so-called corrected Akaike information criterion $\left(\mathrm{AIC}_{\mathrm{C}}\right)$, which is expressed as:

$$
\mathrm{AIC}_{\mathrm{c}}=N \ln \left(\frac{S S E}{N}\right)+2 K+\frac{2 K(K+1)}{N-K-1}
$$

The individual AIC and $\mathrm{AIC}_{\mathrm{c}}$ scores lack universal comparability, as they are influenced by sample size, for instance, and must be converted for a meaningful interpretation of model plausibility as follows:

$$
\Delta_{i}=\mathrm{AIC}_{\mathrm{c}_{i}}-\mathrm{AIC}_{\mathrm{c}_{\text {min }}}
$$

where $\Delta_{i}$ is the information loss occurring if model $i$ is selected rather than the best-fit model, $i_{\text {min }}$, for conclusions. $\mathrm{AIC}_{\mathrm{c} i}$ and $\mathrm{AIC}_{\mathrm{cmin}}$ present the $\mathrm{AIC}_{\mathrm{c}}$ value for model $i$ and the minimum $\mathrm{AIC}_{\mathrm{c}}$ value of all models studied, respectively. Hence, the best approximating model yields $\Delta_{i}=0$ and with increasing $\Delta_{i}$, the strength of evidence for the respective model decreases. The transformation $L$ (model/data) $\propto \exp \left(-\frac{1}{2} \Delta_{i}\right)$ facilitates an estimation of the likelihood of model $i$, where $\exp \left(-\frac{1}{2} \Delta_{i}\right)$ is the relative likelihood of model $i$, and therefore permits an evaluation of the probability of a model being the most approximating among all candidates. The normalization of the relative likelihoods yields the Akaike weight, reflecting the "weight of evidence" in support of a model to be the best fitting, and is defined as:

$$
w_{i}=\frac{e^{\left(-\frac{1}{2} \Delta_{i}\right)}}{\sum_{i=1}^{R} e^{\left(-\frac{1}{2} \Delta_{i}\right)}}
$$

where $w_{i}$ is the Akaike weight of the $i$ th model [28].

Additionally, the evidence ratio $(E R)$ quantifies how much more likely the best approximating model is than model $i$, and is given by:

$$
E R_{i}=\frac{w_{\text {best }}}{w_{i}}
$$

where $w_{\text {best }}$ is the Akaike weight of the best model [28].

\subsection{External Mass Transfer Model (EMTM)}

In-depth investigations of the adsorption kinetics were conducted to elucidate the governing mechanism and characteristics of the adsorption processes studied. We applied the external mass transfer model (EMTM) to describe the decay curves of TMP and ATN adsorption on PPH [21]. The EMTM supposes that the rate of adsorption is limited solely by the external mass transport through the boundary layer. The model assumes the absence of a concentration gradient inside the particle, and therefore that intraparticle diffusion occurs instantaneous. Hence, the model excludes the presence of an internal mass transfer resistance. The EMTM and the initial and boundary conditions [29] are given by:

$$
\begin{gathered}
\frac{d c}{d t}=-A_{S} k_{F}\left(c-c_{r}\right) \\
t=0, c=c_{0}
\end{gathered}
$$




$$
\begin{gathered}
\frac{\varepsilon_{p}}{\rho_{p}} \frac{\partial c_{r}}{\partial t}+\frac{\partial q}{\partial t}=\frac{A_{S} k_{F}}{m}\left(c-c_{r}\right) \\
t=0, c_{r}=0, q=0 .
\end{gathered}
$$

Here, $c$ and $q$ are the adsorbate concentration in aqueous solution $(\mu \mathrm{g} / \mathrm{L})$ and on the adsorbent $(\mu \mathrm{g} / \mathrm{g})$, respectively; $c_{r}$ is the adsorbate concentration inside the particle $(\mu \mathrm{g} / \mathrm{L})$; $m$ is the mass of the adsorbent $(\mathrm{g}) ; A_{S}$ is the total external surface for mass transfer $(1 / \mathrm{cm})$; $\varepsilon_{p}$ is the adsorbent particle porosity; $\rho_{p}$ is the apparent density of the adsorbent $\left(\mathrm{g} / \mathrm{cm}^{3}\right) ; k_{F}$ is the external mass transfer coefficient through the boundary layer $(\mathrm{cm} / \mathrm{s})$.

The total external surface of the adsorbent particle available for mass transfer resulted from:

$$
A_{S}=\frac{6 M}{d_{p} \rho_{p}\left(1-\varepsilon_{p}\right)},
$$

where $M$ is the adsorbent mass concentration in solution $(\mathrm{g} / \mathrm{mL})$ and $d_{p}$ is the adsorbent particle diameter $(\mathrm{cm})$ [47].

From Equation (3), the derivative in time of $q$ becomes:

$$
\frac{\partial q}{\partial t}=\frac{\partial q}{\partial c_{r}} \frac{\partial c_{r}}{\partial t}=K_{H} \frac{\partial c_{r}}{\partial t}
$$

therefore, Equation (15) above can be rewritten as:

$$
\left(\frac{\varepsilon_{p}}{\rho_{p}}+K_{H}\right) \frac{\partial c_{r}}{\partial t}=A_{S} k_{F}\left(c-c_{r}\right) .
$$

In this case, the ordinary differential equations of the EMTM can be exactly solved with the given initial conditions. The concentration in aqueous solution changes with time as:

$$
c(t)=c_{0} \frac{\left(1+e^{\left.-\mathrm{A}_{\mathrm{s}} \mathrm{k}_{\mathrm{F}} t\left(\frac{\mathrm{M} \lambda+1}{\mathrm{M} \lambda}\right) M \lambda\right)}\right.}{1+M \lambda},
$$

where

$$
\lambda=\left(\frac{\varepsilon_{p}}{\rho_{p}}+K_{H}\right) .
$$

The final $k_{F}$ values were determined by minimizing the nonlinear least squares objective function between predicted data $\left(c_{t, c a l}\right)$ from Equation (20) and experimental data $\left(c_{t, \text { exp }}\right)$ of TMP or ATN concentrations and were calculated analogous to Equation (6).

\subsection{Adsorption Thermodynamics}

Detailed investigations of the adsorption thermodynamics are crucial to derive information on the nature and mechanism of the adsorption process. The relevant thermodynamic parameters considered for this evaluation, i.e., the standard free Gibbs energy $\left(\Delta G^{\circ}\right)$, standard enthalpy $\left(\Delta H^{\circ}\right)$, and standard entropy $\left(\Delta S^{\circ}\right)$, were computed according to following equations $[18,19]$ :

$$
\Delta G^{\circ}=-R T \ln \left(K_{e q}\right),
$$

where $R$ is the universal gas constant $(8.3145 \mathrm{~J} / \mathrm{mol} \mathrm{K}), T$ is the temperature $(\mathrm{K})$, and $K_{\text {eq }}$ represents the thermodynamic equilibrium constant (-). $\Delta G^{\circ}$ was derived directly from Equation (22). The relationship of $\Delta G^{\circ}$ with $\Delta H^{\circ}$ and $\Delta S^{\circ}$ is given by:

$$
\Delta G^{\circ}=\Delta H^{\circ}-\mathrm{T} \Delta S^{\circ} .
$$

Substitution of Equation (22) into Equation (23) results in the Van't Hoff equation, which is expressed as:

$$
\ln \left(K_{e q}\right)=\frac{\Delta S^{\circ}}{R}-\frac{\Delta H^{\circ}}{R T} .
$$


The plot of $\ln K_{e q}$ versus $10^{3} / T$, known as the Van't Hoff plot, facilitates the determination of $\Delta H^{\circ}$ and $\Delta S^{\circ}$ from the slope and intercept, respectively [29]. A correct calculation of $K_{e q}$ is imperative for a meaningful estimation of the thermodynamic parameters. The dimensionality and logarithmic computation in Equations (22) and (24) require $K_{e q}$ to be dimensionless $[18,19]$. In case the equilibrium data is described by the Henry isotherm, the thermodynamic equilibrium $K_{e q}$ constant may be obtained from the isotherm constant $K_{H}$ after appropriate conversion. The conversion of $K_{H}$ allows omitting the dimensions and is given by:

$$
K_{e q} \approx K_{H} \rho_{W}(T),
$$

where $\rho_{W}$ is the density of water $(\mathrm{g} / \mathrm{L})$ at temperature $T$ [19].

\subsection{Statistical Analysis}

All experiments were conducted in triplicate and the data were expressed as mean values \pm standard deviation unless otherwise stated. One-way analysis of variance (ANOVA) with Tukey's post-hoc test was carried out to compare mean group values. An alpha value of 0.05 was considered to be significant. The data was analyzed using Origin, Version 2021 (OriginLab Corporation, Northampton, MA, USA).

\section{Conclusions}

Pigeon pea husk (PPH) demonstrated encouraging properties as a low-cost adsorbent for the removal of two abundantly occurring pharmaceuticals in Indian water bodies, namely trimethoprim (TMP) and atenolol (ATN). PPH exhibited a linear increase in adsorption capacity upon increasing concentrations of TMP and ATN considering the order of magnitude of actual concentrations of these pharmaceuticals found in Indian waters. Additionally, the description of the saturation limit with an adequate isotherm model, selected based on the AIC criterion, validated the technological suitability of PPH for the studied adsorbates likewise at a higher concentration. The investigations of the adsorption kinetics using the EMTM revealed coherently that film diffusion posed the exclusive mass transfer resistance of TMP or ATN adsorption onto PPH. Furthermore, thermodynamic elucidations unveiled weak molecular interactions as driving mechanisms for both adsorption systems studied, and hold promise for future work on the regeneration of $\mathrm{PPH}$ and the desorption of the adsorbates. The highest adsorption loadings achieved for TMP and ATN concur with the $\mathrm{pH}$ range reported for Indian water bodies, substantiating PPH as a viable asset for low-cost adsorption of the considered pharmaceuticals. This study provides proof of concept of the merits of PPH as a low-cost adsorbent for TMP and ATN and depicts the promising prospect of extending its application to contaminants with similar characteristics. Ultimately, our findings depict the scientific foundation for future work on multicomponent systems, the investigation of a column adsorber design for practical applications, and the reusability of PPH aiming at the development of a viable adsorber system for low-cost water treatment.

Supplementary Materials: The following are available online, Figure S1: Particle size distribution of PPH, Table S1: Effect of solution temperature on adsorption capacity of PPH for TMP or ATN. Different letters denote significant differences between isotherm points $(p<0.05, n=3)$, Figure S2: Determination of point of zero charge of PPH. Description of adsorption isotherm models.

Author Contributions: Conceptualization, S.E.; methodology, S.E.; formal analysis, S.E., M.T., and P.A.; investigation, S.E., A.M., and M.T.; resources, L.N.; writing—original draft preparation, S.E., A.M., M.T., and P.A.; writing—review and editing, S.E., A.M., M.T., P.A., and L.N.; visualization, S.E.; supervision, S.E. and L.N.; project administration, L.N.; funding acquisition, L.N. All authors have read and agreed to the published version of the manuscript.

Funding: This research was supported by the European Research Council ERC, under the European Union's Horizon 2020 research and innovation programme (grant agreement no. 679037), and ETH Zurich. 
Institutional Review Board Statement: Not applicable.

Informed Consent Statement: Not applicable.

Data Availability Statement: The data presented in this study are available on request from the corresponding author.

Acknowledgments: The authors acknowledge Angus E. McMillan from the Laboratory for organic chemistry for his support and guidance in the acquisition of the FTIR spectra. The authors gratefully thank Anne Greet Bittermann of Scope M, ETH Zürich, for supporting the SEM analysis and sharing her expertise. The authors gratefully acknowledge Michael Plötze from the Claylab, ETH Zürich for his help and assistance in the physical characterization of the adsorbent material. The authors are thankful to Bühler AG (Uzwil, Switzerland) for providing the raw pigeon pea husk material.

Conflicts of Interest: The authors declare no conflict of interest.

Sample Availability: Samples of the compounds are available from the authors.

\section{References}

1. Bhatnagar, A.; Sillanpää, M. Utilization of agro-industrial and municipal waste materials as potential adsorbents for water treatment-A review. Chem. Eng. J. 2010, 157, 277-296. [CrossRef]

2. De Gisi, S.; Lofrano, G.; Grassi, M.; Notarnicola, M. Characteristics and adsorption capacities of low-cost sorbents for wastewater treatment: A review. Sustain. Mater. Technol. 2016, 9, 10-40. [CrossRef]

3. Gani Khalid, M.; Kazmi Absar, A. Contamination of Emerging Contaminants in Indian Aquatic Sources: First Overview of the Situation. J. Hazard. ToxicRadioact. Waste 2017, 21, 04016026. [CrossRef]

4. Grassi, M.; Kaykioglu, G.; Belgiorno, V.; Lofrano, G. Removal of Emerging Contaminants from Water and Wastewater by Adsorption Process. In Emerging Compounds Removal from Wastewater: Natural and Solar Based Treatments; Lofrano, G., Ed.; Springer: Dordrecht, The Netherland, 2012; pp. 15-37.

5. Philip, J.M.; Aravind, U.K.; Aravindakumar, C.T. Emerging contaminants in Indian environmental matrices-A review. Chemosphere 2018, 190, 307-326. [CrossRef] [PubMed]

6. Balakrishna, K.; Rath, A.; Praveenkumarreddy, Y.; Guruge, K.S.; Subedi, B. A review of the occurrence of pharmaceuticals and personal care products in Indian water bodies. Ecotoxicol. Environ. Saf. 2017, 137, 113-120. [CrossRef]

7. Kraupner, N.; Ebmeyer, S.; Hutinel, M.; Fick, J.; Flach, C.F.; Larsson, D.G.J. Selective concentrations for trimethoprim resistance in aquatic environments. Environ. Int. 2020, 144, 106083. [CrossRef]

8. Steinbach, C.; Burkina, V.; Fedorova, G.; Grabicova, K.; Stara, A.; Velisek, J.; Zlabek, V.; Schmidt-Posthaus, H.; Grabic, R.; Kocour Kroupova, $\mathrm{H}$. The sub-lethal effects and tissue concentration of the human pharmaceutical atenolol in rainbow trout (Oncorhynchus mykiss). Sci. Total Environ. 2014, 497-498, 209-218. [CrossRef]

9. Singh, K.P.; Rai, P.; Singh, A.K.; Verma, P.; Gupta, S. Occurrence of pharmaceuticals in urban wastewater of north Indian cities and risk assessment. Environ. Monit. Assess. 2014, 186, 6663-6682. [CrossRef]

10. Foo, K.Y.; Hameed, B.H. Insights into the modeling of adsorption isotherm systems. Chem. Eng. J. 2010, 156, 2-10. [CrossRef]

11. Parate, V.R.; Talib, M.I. Utilization of Pulse Processing Waste (Cajanus cajan Husk) for Developing Metal Adsorbent: A Value-added Exploitation of Food Industry Waste. Am. J. Food Sci. Technol. 2015, 3, 1-9.

12. Hang, C.C.; Garnsey, E.; Ruan, Y. Opportunities for disruption. Technovation 2015, 39-40, 83-93. [CrossRef]

13. Saxena, K.B.; Kumar, R.V.; Sultana, R. Quality nutrition through pigeonpea-A review. Health 2010, 2, 1335-1344. [CrossRef]

14. Sarkar, S.; Panda, S.; Yadav, K.K.; Kandasamy, P. Pigeon pea (Cajanus cajan) an important food legume in Indian scenario-A review. Legume Res. Int. J. 2018, 43, 601-610. [CrossRef]

15. Aravind, J.; Muthusamy, S.; Sunderraj, S.H.; Chandran, L.; Palanisamy, K. Pigeon pea (Cajanus cajan) pod as a novel eco-friendly biosorbent: A study on equilibrium and kinetics of $\mathrm{Ni}$ (II) biosorption. Int. J. Ind. Chem. 2013, 4, 25. [CrossRef]

16. Venkata Ramana, D.K.; Harikishore Kumar Reddy, D.; Yu, J.S.; Seshaiah, K. Pigeon peas hulls waste as potential adsorbent for removal of $\mathrm{Pb}$ (II) and $\mathrm{Ni}$ (II) from water. Chem. Eng. J. 2012, 197, 24-33. [CrossRef]

17. Aravind, J.; Lenin, C.; Nancyflavia, C.; Rashika, P.; Saravanan, S. Response surface methodology optimization of nickel (II) removal using pigeon pea pod biosorbent. Int. J. Environ. Sci. Technol. 2015, 12, 105-114. [CrossRef]

18. Tran, H.N.; You, S.-J.; Hosseini-Bandegharaei, A.; Chao, H.-P. Mistakes and inconsistencies regarding adsorption of contaminants from aqueous solutions: A critical review. Water Res. 2017, 120, 88-116. [CrossRef]

19. Ghosal, P.S.; Gupta, A.K. An insight into thermodynamics of adsorptive removal of fluoride by calcined $\mathrm{Ca}-\mathrm{Al}-\left(\mathrm{NO}_{3}\right)$ layered double hydroxide. RSC Adv. 2015, 5, 105889-105900. [CrossRef]

20. Spiess, A.-N.; Neumeyer, N. An evaluation of R2 as an inadequate measure for nonlinear models in pharmacological and biochemical research: A Monte Carlo approach. BMC Pharm. 2010, 10, 6. [CrossRef]

21. Dotto, G.L.; Salau, N.P.G.; Piccin, J.S.; Cadaval, T.R.S.A.; de Pinto, L.A.A. Adsorption Kinetics in Liquid Phase: Modeling for Discontinuous and Continuous Systems. In Adsorption Processes for Water Treatment and Purification; Bonilla-Petriciolet, A., Mendoza-Castillo, D.I., Reynel-Ávila, H.E., Eds.; Springer International Publishing: Cham, Switzerland, 2017 ; pp. 53-76. 
22. Coates, J. Interpretation of Infrared Spectra, A Practical Approach. Encycl. Anal. Chem. 2006. [CrossRef]

23. Manrique, G.D.; Lajolo, F.M. FT-IR spectroscopy as a tool for measuring degree of methyl esterification in pectins isolated from ripening papaya fruit. Postharvest. Biol. Technol. 2002, 25, 99-107. [CrossRef]

24. Leal, D.; Matsuhiro, B.; Rossi, M.; Caruso, F. FT-IR spectra of alginic acid block fractions in three species of brown seaweeds. Carbohydr. Res. 2008, 343, 308-316. [CrossRef] [PubMed]

25. Tella, A.C.; Eke, U.B.; Owalude, S.O. Solvent-free mechanochemical synthesis and X-ray studies of Cu(II) and Ni(II) complexes of 5-(3,4,5-Trimethoxybenzyl)pyrimidine-2,4-diamine (Trimethoprim) in a ball-mill. J. Saudi Chem. Soc. 2016, 20, S376-S381. [CrossRef]

26. Djellouli, F.; Dahmani, A.; Hassani, A. Characterization of the polymorph changes in Trimethoprim. J. Therm. Anal. Calorim. 2017, 130, 1585-1591. [CrossRef]

27. Giles, C.H.; MacEwan, T.H.; Nakhwa, S.N.; Smith, D. Studies in adsorption. Part XI. A system of classification of solution adsorption isotherms, and its use in diagnosis of adsorption mechanisms and in measurement of specific surface areas of solids. $J$. Chem. Soc. (Resumed) 1960, 786, 3973-3993. [CrossRef]

28. Burnham, K.P.; Anderson, D.R. Multimodel inference: Understanding AIC and BIC in model selection. Sociol. Methods Res. 2004, 33, 261-304. [CrossRef]

29. Piccin, J.S.; Cadaval, T.R.S.A.; de Pinto, L.A.A.; Dotto, G.L. Adsorption Isotherms in Liquid Phase: Experimental, Modeling, and Interpretations. In Adsorption Processes for Water Treatment and Purification; Bonilla-Petriciolet, A., Mendoza-Castillo, D.I., Reynel-Ávila, H.E., Eds.; Springer International Publishing: Cham, Switzerland, 2017; pp. 19-51.

30. Worch, E. Adsorption equilibrium I: General aspects and single-solute adsorption. In Adsorption Technology in Water Treatment, Fundamentals, Processes, and Modeling; De Gruyter: Berlin, Germany; Boston, FL, USA, 2012; pp. 41-76.

31. Li, Y.; Taggart, M.A.; McKenzie, C.; Zhang, Z.; Lu, Y.; Pap, S.; Gibb, S. Utilizing low-cost natural waste for the removal of pharmaceuticals from water: Mechanisms, isotherms and kinetics at low concentrations. J. Clean. Prod. 2019, 227, 88-97. [CrossRef]

32. Nielsen, L.; Bandosz, T.J. Analysis of sulfamethoxazole and trimethoprim adsorption on sewage sludge and fish waste derived adsorbents. Microporous Mesoporous Mater. 2016, 220, 58-72. [CrossRef]

33. Bekçi, Z.; Seki, Y.; Yurdakoç, M.K. Equilibrium studies for trimethoprim adsorption on montmorillonite KSF. J. Hazard. Mater. 2006, 133, 233-242. [CrossRef]

34. Hu, Y.; Fitzgerald, N.M.; Lv, G.; Xing, X.; Jiang, W.-T.; Li, Z. Adsorption of Atenolol on Kaolinite. Adv. Mater. Sci. Eng. 2015, 2015, 897870. [CrossRef]

35. Haro, N.K.; Del Vecchio, P.; Marcilio, N.R.; Féris, L.A. Removal of atenolol by adsorption—Study of kinetics and equilibrium. J. Clean. Prod. 2017, 154, 214-219. [CrossRef]

36. To, M.-H.; Hadi, P.; Hui, C.-W.; Lin, C.S.K.; McKay, G. Mechanistic study of atenolol, acebutolol and carbamazepine adsorption on waste biomass derived activated carbon. J. Mol. Liq. 2017, 241, 386-398. [CrossRef]

37. Worch, E. Adsorption kinetics. In Adsorption Technology in Water Treatment, Fundamentals, Processes, and Modeling; De Gruyter: Berlin, Germany; Boston, FL, USA, 2012; pp. 123-168.

38. Saha, P.; Chowdhury, S. Insight Into Adsorption Thermodynamics. In Thermodynamics; Tadashi, M., Ed.; IntechOpen: London, UK, 2011; pp. 349-364.

39. Wang, P.; He, Y.-L.; Huang, C.-H. Oxidation of Antibiotic Agent Trimethoprim by Chlorine Dioxide: Reaction Kinetics and Pathways. J. Environ. Eng. 2012, 138, 360-366. [CrossRef]

40. Martínez, V.; Maguregui, M.I.; Jiménez, R.M.; Alonso, R.M. Determination of the pKa values of $\beta$-blockers by automated potentiometric titrations. J. Pharm. Biomed. Anal. 2000, 23, 459-468. [CrossRef]

41. Avvannavar, S.M.; Shrihari, S. Evaluation of water quality index for drinking purposes for river Netravathi, Mangalore, South India. Environ. Monit. Assess. 2008, 143, 279-290. [CrossRef]

42. Bhardwaj, R.M. Water Quality Monitoring In India- Achievements and Constraints. Int. J. Inf. Comput. Technol. 2005, 3, 851-856.

43. Dash, A.K. Characterization of Domestic Wastewater at Bhubaneswar, Odisha, India. Development 2013, 25, 27.

44. Souza, P.R.; Dotto, G.L.; Salau, N.P.G. Detailed numerical solution of pore volume and surface diffusion model in adsorption systems. Chem. Eng. Res. Des. 2017, 122, 298-307. [CrossRef]

45. Krisko, R.M.; McLaughlin, K.; Koenigbauer, M.J.; Lunte, C.E. Application of a column selection system and DryLab software for high-performance liquid chromatography method development. J. Chromatogr. A 2006, 1122, 186-193. [CrossRef]

46. Sugiura, N. Further analysts of the data by akaike's information criterion and the finite corrections. Commun. Stat. Theory Methods 1978, 7, 13-26. [CrossRef]

47. Furusawa, T.; Smith, J.M. Fluid-Particle and Intraparticle Mass Transport Rates in Slurries. Ind. Eng. Chem. Fundam. 1973, 12, 197-203. [CrossRef] 